\title{
Comparison of PD-L1 immunohistochemical assays and the significance of PD-L1 expression in thymoma
}

\author{
Shintaro Yokoyama ${ }^{1}$, Hiroaki Miyoshi ${ }^{2}$ \\ ${ }^{1}$ Department of Surgery, Kurume University School of Medicine, Kurume, Japan; ${ }^{2}$ Department of Pathology, Kurume University School of \\ Medicine, Kurume, Japan \\ Contributions: (I) Conception and design: All authors; (II) Administrative support: None; (III) Provision of study materials or patients: All authors; (IV) \\ Collection and assembly of data: S Yokoyama; (V) Data analysis and interpretation: S Yokoyama; (VI) Manuscript writing: All authors; (VII) Final \\ approval of manuscript: All authors. \\ Correspondence to: Hiroaki Miyoshi, MD, PhD, Department of Pathology, Kurume University School of Medicine, 67 Asahi-machi, Kurume, \\ 8300011, Japan. Email: miyoshi_hiroaki@med.kurume-u.ac.jp.
}

\begin{abstract}
Thymoma is a relatively rare malignancy, which is categorized as thymic epithelial tumor but known as the most common pathology that is developed in the anterior mediastinum. Complete resection is recommended for localized tumors and usually favorable prognosis can be obtained. However, poor survival period has been reported in unresectable cases exhibiting extensive invasion or distant metastasis, as effective chemotherapeutic regimens are restrained. We previously assessed expression of programmed death ligand 1 (PD-L1) and programmed death 1 (PD-1) and discussed their prospective application in the immunotherapy of thymic epithelial tumors. After our publication, additional studies using reliable PD-L1 antibodies, which are currently administered to predict efficacy of PD-1/PD-L1 blockade therapy were performed and further characterized PD-L1 in thymoma. Herein, recent knowledge in relation to the significance of PD-L1 expression in thymoma is reviewed based on recent findings using qualified PD-L1 clones. Most studies coherently found high expression of PD-L1 on the cell membrane and cytoplasm of tumor epithelial cells in accordance with previous reports, which is a predictive marker for effectiveness of anti-PD-1/PD-L1 drugs, even when approved PD-L1 antibodies were employed. On the other hand, PD-L1 expression on tumor infiltrating immune cells remains to be sufficiently determined. High PD-L1 expression can be expected in cases with high grade histological subtypes, such as type B2/B3 thymomas, or those with advanced stages III or IV of the disease. Interestingly, the level of PD-L1 expression was found to be upregulated after chemotherapy compared with that before, which could be explained by immunogenic cell death. The prognostic impact of PD-L1 expression in thymoma might be found only when thymic carcinoma patients were excluded. Furthermore, it also could be identified when we analyzed thymomas completely resected, distinct from biopsy and incompletely resected cases.
\end{abstract}

Keywords: Thymoma; tumor immunology; programmed death ligand 1 (PD-L1)

Submitted Jan 15, 2020. Accepted for publication May 13, 2020.

doi: $10.21037 /$ jtd-19-3703

View this article at: http://dx.doi.org/10.21037/jtd-19-3703

\section{Introduction}

Thymoma is an uncommon malignant neoplasm that arises in the anterior mediastinum. It is widely known to originate from thymic epithelial cells, with a unique ability to partly promote $\mathrm{T}$ cell maturation from immature progenitors (1). On the basis of a number of retrospective studies, surgical complete resection is currently the mainstay for the treatment of localized thymoma with a high cure rate $(2,3)$. In contrast, the optimal treatment for advanced thymoma has long been debated, and it remains to be clearly defined. For locally advanced thymoma, the National Comprehensive Cancer Network guidelines recommend multimodality therapy including induction chemotherapy 
followed by surgery in unresectable cases, those with solitary metastasis, or those with ipsilateral pleural metastasis (4). Evidence of extrathoracic metastases oblige thymoma patients to opt for chemotherapy. It seems that cisplatin-doxorubicin based regimens yield better outcomes; however, the results are by no means satisfactory (5). The clinical activity of molecular targeted drugs for thymomas has still not been demonstrated, whereas an oral tyrosine kinase inhibitor, sunitinib, seems to be active in thymic carcinoma (6). Therefore, clinicians urgently require an innovative breakthrough to treat thymoma patients with distant metastases based on underlying solid molecular oncogenic mechanisms.

Programmed death ligand 1 (PD-L1) is a transmembrane glycoprotein expressed on antigen-presenting cells and inhibits cytokine production and cell cycle progression in $T$ cells by binding to its receptor of programmed death 1 (PD-1), which is a member of the B7 receptor family expressed on the surface of $\mathrm{T}$ cells $(7,8)$. Notably, PD-1/ PD-L1 pathway is essential not only in normal immune system regulation but also facilitates tumor progression by inhibiting anti-tumor $\mathrm{T}$ cell responses to evade host immune surveillances $(9,10)$. The discovery of this mechanism inspired researchers to access expression levels of PD-L1 or PD-1 and estimate their potential as novel hallmarks to be used as prognostic markers for malignant tumors. In fact, expressions of PD-L1 in tumor cells and of PD-1 in tumor infiltrating immune cells have been reported to predict survival in various malignancies (11-14). PD-L1 expression in thymic epithelial tumors (TETs) has also gradually been characterized by several researchers, whereas obtained findings were slightly different. The authors deem that significance of PD-L1 expression should be carefully argued particularly in thymoma, since it occurs in thymus that is a unique organ to promote $\mathrm{T}$ cell maturation. Indeed, thymoma, in contrast to other malignant tumors, is universally recognized to be infiltrated by immature $\mathrm{T}$ cells positive for terminal deoxynucleotidyl transferase, cluster of differentiation (CD) 1a and CD99 with varied distribution depending on tumor subtypes (15). Consequently, PD-L1 in thymoma may potentially play a role, which is different from the other malignancies.

In 2018, the authors published a review article regarding the condition of PD-L1/PD-1 expression and discussed the perspective of immunotherapy in TETs (16). Some studies subsequently appended observations of labeling against PD-L1 antibodies in TETs, including application of currently administered agents to predict efficacy of PD-1/PD-L1 blockade therapy. These data let us intrinsically understand PD-L1 functions in tumor microenvironment of TETs and argue whether its expression can be a valuable prognostic predictor. Furthermore, analyses of thymomas distinctively from thymic carcinomas in respect of these novel findings could refine the knowledge of PD-L1 specific roles served in thymomas. Taking together, comprehensive PD-L1 analyses in thymomas are reviewed in this article focused on the comparison among various PD-L1 antibodies. Along with the expression patterns, their associations with clinicopathological features and prognostic impact in the tumors are rendered.

\section{Expression patterns, associated properties, and prognostic value of PD-L1 expression in thymoma based on each PD-L1 antibody}

As far as we explored, 15 studies mentioned PD-L1 expression in thymoma. Table 1 summarizes PD-L1 condition and incidence in each study, which include approaches to evaluate PD-L1 abundance and cutoff value determinants. Furthermore, identified factors and prognostic value associated with high PD-L1 expression are also listed in Table 2. The major findings of each study are noted in the following descriptions according to respective PD-L1 antibodies.

\section{Clone 22C3}

PD-L1 expression in 32 thymomas was investigated by Owen et al. via a clone of 22C3 (Merck Research Laboratories, Palo Alto, CA, USA), used for companion diagnostics for pembrolizumab with approval by the US Food and Drug Administration (FDA) in cervical cancer, gastric carcinoma, non-small cell lung cancer, and urothelial carcinoma (17). PD-L1 investigation in thymoma using $22 \mathrm{C} 3$ has been described only in this report. The authors identified high rate as much as $81 \%$ of tumors demonstrating high PD-L1 expression by semiquantitative scoring method. PD-L1 staining seemed to be mainly observed in tumor epithelial cells, whereas staining on immune cells was not clearly described. In addition, apparent intratumoral heterogeneity of PD-L1 expression in some cases was remarkable, similarly to the study in nonsmall cell lung cancer (31). This is the only report that decidedly identified intratumoral heterogeneity of PD-L1 in thymoma, which was observed in $\mathrm{AB}$ thymoma as well as B3 thymoma with minor B2 component. As thymomas 
Table 1 PD-L1 expression status according to PD-L1 antibodies in thymoma

\begin{tabular}{|c|c|c|c|c|c|c|}
\hline PD-L1 antibody & FDA approval & Study & Year & $\begin{array}{c}\text { Thymoma, } \\
\mathrm{n}\end{array}$ & $\begin{array}{l}\text { High PD-L1 } \\
\text { expression (\%) }\end{array}$ & PD-L1 cutoff value \\
\hline $22 \mathrm{C} 3$ & $\begin{array}{l}\text { Companion diagnostic } \\
\text { for pembrolizumab }\end{array}$ & Owen et al. (17) & 2018 & 32 & 81 & $\begin{array}{l}\text { Semiquantitative scoring (high or } \\
\text { moderate) }\end{array}$ \\
\hline \multirow[t]{2}{*}{ SP142 } & \multirow{2}{*}{$\begin{array}{l}\text { Companion and } \\
\text { complementary } \\
\text { diagnostics for } \\
\text { atezolizumab }\end{array}$} & Marchevsky et al. (18) & 2017 & 38 & 92 & Staining area $(1 \%)$ \\
\hline & & Chen et al. (19) & 2018 & 50 & 48 & Quantitative, H-score (3\%) \\
\hline \multirow[t]{2}{*}{ SP263 } & \multirow{2}{*}{$\begin{array}{l}\text { Complementary } \\
\text { diagnostic for } \\
\text { durvalumab }\end{array}$} & Guleria et al. (21) & 2018 & 84 & 82 & Staining area $(25 \%)$ \\
\hline & & Terra et al. (22) & 2019 & 11 & $\begin{array}{l}91 \text { (cutoff } 1 \%) \text {, } \\
82 \text { (cut off } 50 \% \text { ) }\end{array}$ & Number of cells ( $1 \%$ and $50 \%)$ \\
\hline \multirow[t]{2}{*}{ E1L3N } & \multirow[t]{2}{*}{-} & Katsuya et al. (23) & 2015 & 101 & 23 & Quantitative, H-score $(3 ; 1 \%)$ \\
\hline & & Tiseo et al. (26) & 2017 & 87 & 18 & Quantitative, H-score $(3 ; 1 \%)$ \\
\hline Other antibody & - & & & & & \\
\hline 29E.2A3, 29E.5A9 & & Brown et al. (8) & 2003 & 26 & 81 & NA \\
\hline 15 & & Padda et al. (27) & 2015 & 65 & 68 & $\begin{array}{l}\text { Intensity (intermediate-strong } \\
\text { staining) }\end{array}$ \\
\hline EPR1161[2] & & Yokoyama et al. (28) & 2016 & 82 & 54 & Staining area $(38 \%)$ \\
\hline $\begin{array}{l}\text { ab58810 (catalog } \\
\text { number) }\end{array}$ & & Duan et al. (29) & 2018 & 13 & 46 & Quantitative (median value) \\
\hline
\end{tabular}

PD-L1, programmed death ligand 1; FDA, Food and Drug Administration; NA, not available.

sometimes contain heterogeneous histologic components, this appraisal can be rational. Any further association between high PD-L1 expression and clinicopathological features, including World Health Organization (WHO) histological subtype and tumor stage, was not found. As for prognosis, PD-L1 positivity did not demonstrate statistical significance both in overall or event-free survival even when 3 thymic carcinoma samples were excluded, although PD-L1 positive thymomas tended to reveal worse survival rather than negative.

\section{Clone SP142}

A PD-L1 antibody of SP142 (Spring Bioscience, Pleasanton, CA, USA and Ventana Medical Systems, Tucson, AZ, USA) was employed by 3 authors (18-20). This antibody has been approved by FDA for companion diagnostic for atezolizumab in triple negative breast carcinoma, as well as complementary diagnostic in nonsmall cell lung cancer and urothelial carcinoma. It seems that all authors are accordant that PD-L1 was profusely expressed in tumor epithelial cells; nevertheless, the population of PD-L1 positive cases varied among studies ranged between $27 \%$ to $92 \%$. This is may be due to distinct cutoff values and evaluation methods, meaning whether both or either staining intensity and propensity are taken into consideration. In addition, altered tumor profiles such as histological subtypes, tumor staging, or prior treatments may be the influencing factors. Moreover, two authors using this clone notably alluded that PD-L1 was also expressed in tumor infiltrating lymphocytes and macrophages. In fact, PD-L1 expression on tumor infiltrating immune cells has been reported in some malignant tumors to be a favorable prognostic predictor, although detailed mechanisms still 
Table 2 Significance of high PD-L1 expression on the basis of PD-L1 antibodies in thymoma

\begin{tabular}{|c|c|c|c|}
\hline PD-L1 antibody & Study & $\begin{array}{l}\text { Profiles associated with high PD-L1 } \\
\text { expression }\end{array}$ & $\begin{array}{l}\text { Prognostic value of high PD-L1 } \\
\text { expression }\end{array}$ \\
\hline $22 \mathrm{C} 3$ & Owen et al. (17) & None* $^{*}$ & None \\
\hline \multirow[t]{2}{*}{ SP142 } & Marchevsky et al. (18) & WHO type B2, B3 & NA \\
\hline & Chen et al. (19) & $\begin{array}{l}\text { WHO classification, Masaoka-Koga staging, } \\
\text { radiotherapy, chemotherapy* }\end{array}$ & NA \\
\hline \multirow[t]{2}{*}{ SP263 } & Guleria et al. (21) & Type B tumors (B1, B2, B3) & None \\
\hline & Terra et al. (22) & NA & NA \\
\hline \multirow[t]{2}{*}{ E1L3N } & Katsuya et al. (23) & None ${ }^{*}$ & None \\
\hline & Tiseo et al. (26) & Masaoka-Koga stage $^{\star}$ & None \\
\hline \multicolumn{4}{|l|}{ Other antibody } \\
\hline 29E.2A3, 29E.5A9 & Brown et al. (8) & NA & NA \\
\hline 15 & Padda et al. (27) & $\begin{array}{l}\text { Younger age, higher stage, incomplete } \\
\text { resection, WHO classification* }\end{array}$ & $\begin{array}{l}\text { Worse OS (age- and sex-adjusted } \\
\text { analysis)* }\end{array}$ \\
\hline EPR1161[2] & Yokoyama et al. (28) & WHO type B2/B3, Masaoka stage III/IV & Worse DFS \\
\hline ab58810 (catalog number) & Duan et al. (29) & None $^{*}$ & None \\
\hline $27 \mathrm{~A} 2$ & Bagir et al. (30) & None* & None* \\
\hline
\end{tabular}

*, including cases of thymic carcinoma. PD-L1, programmed death ligand 1; WHO, World Health Organization; NA, not available; SUVmax, maximum standardized uptake value; FDG-PET, fluorine-18 fluorodeoxyglucose positron emission tomography; OS, overall survival; DFS, disease-free survival.

remain uncertain $(32,33)$. For the further assessment of PD-L1 within tumor microenvironment, it is pivotal to clarify whether tumor infiltrating immune cells indeed express PD-L1 in thymoma. All authors found PD-L1 to be higher expressed in type B2/B3 thymoma compared with the other subtypes. Clinical staging also influenced PD-L1 abundance, demonstrating that high expression was confirmed in advanced tumors. Chen et al. (19) reported that radiotherapy and chemotherapy as received treatments were associated with high PD-L1 expression, although this analysis was performed using a cohort including thymic carcinomas. Hakiri et al. (20) found that high PD-L1 expression was significantly correlated with tumors showing high standardized uptake value on fluorine- 18 fluorodeoxyglucose positron emission tomography, which can be utilized in clinical settings. Among studies, prognostic implications were examined only by Hakiri et al., demonstrating that cases expressing high levels of PD-L1 were significantly associated with worse overall survival. In contrast to the other studies which include patients who underwent only diagnostic biopsy, the patient group of Hakiri et al. included high proportion of patients following surgical complete resection, which can elicit substantial survival relevance of PD-L1 expression in thymoma.

\section{Clone SP263}

SP263 (Ventana Medical Systems) is an antibody approved by FDA for complementary diagnostic for durvalumab in urothelial carcinoma. Guleria et al. used this clone and detected $82 \%$ of thymoma, classified as high-level PD-L1 expression using cutoff of staining area more than $25 \%$ (21). This observation was supported by Terra $e t$ al. finding that 91\% of thymoma to be categorized as having high PD-L1 
expression when $1 \%$ of number of cells was employed for the cutoff value (22). The latter also determined as much as $82 \%$ of thymomas were still classified as positive even when the number of cells stained more than $50 \%$ was used as the cutoff value. Despite higher PD-L1 cutoff value definition compared with other studies, both of them found considerably high rate of PD-L1 expression. This suggests that SP263 may indicate strong positivity as compared with other PD-L1 antibodies, although potential false-positive cases still remain unknown. Both studies did not find PDL1 expression on tumor infiltrating immune cells. Guleria et al. identified type B tumors associated with high PD-L1 expression, which is in accordance with earlier descriptions of SP142 finding that type B2/B3 thymoma showed high PD-L1 expression. Prognostic values were also analyzed by them, concluding that any impact could not be determined.

\section{Clone E1L3N}

A clone of E1L3N (Cell Signaling Technology, Danvers, MA, USA) is recognized as a qualified PD-L1 antibody, which was confirmed by a comparative study in nonsmall cell lung cancer (34). This antibody was adopted in thymoma by 4 different groups (23-26). Katsuya et al. and Tiseo et al. employed the same evaluation method (H-score) and cutoff value for PD-L1 definition. As a result, they showed similarity in the proportion of high-level PD-L1 expression cases as $20 \%$. By contrast, the other two authors revealed higher population of $\mathrm{PD}-\mathrm{L} 1$ positive cases. All studies are in concordance that PD-L1 expression was observed only in epithelial cells in thymoma but not in infiltrating immune cells.

Of note, Weissferdt et al. reported an important observation that PD-L1 was overexpressed in cases with neoadjuvant chemotherapy, as shown above by Chen et al. using SP142. The result was similarly demonstrated by Katsuya et al. with sequential surveillance (35). They compared PD-L1 expression positivity of thymoma tissue specimens obtained before and after chemotherapy, and discovered that PD-L1 expression after chemotherapy was increased in all cases. This agreement could be explained by immunogenic cell death of tumor cells after chemotherapy. The immunogenic cell death is obviously known to be occurred through the use of some chemotherapeutic agents (36). The activation of dendritic cells was enhanced by immunogenic cell death, following activated $\mathrm{T}$ cells to produce interferon-gamma which induces PD-L1 expression on the tumor cells (37). Further clarifications with respect to molecular mechanisms of PD-L1 should enhance understanding the interactions within immunological microenvironment in thymoma, being beneficial as a hint for development of effective managements in the treatment of this rare malignancy.

On the other hand, prognostic value of PD-L1 appeared not to be significant in the studies using this antibody. One study determined its significance as a better prognostic factor; however, this may be potentially biased due to collective analysis including thymoma and thymic carcinoma.

\section{Other PD-L1 antibodies}

PD-L1 expression on thymic gland was primarily presented by Brown et al. using normal fetal thymus (8). This group claimed that PD-L1 expression pattern stained with the self-generated clones (29E.2A3 and 29E.5A9) in thymic tissue coincided with the staining pattern of anticytokeratin monoclonal antibody specific for epithelial cell markers, suggesting that PD-L1 was expressed on thymic epithelial cells. After a certain period, Padda et al. reported diffuse high intensity of PD-L1 expression in TETs (27). They found that $68 \%$ of thymomas showed high PD-L1 expression using a clone of 15 (Sino Biological, Beijing, China). PD-L1 positivity was defined based on the intensity of intermediate-strong staining in epithelial cells as criteria for high expression. It was found that PD-L1 is also expressed in tumor-associated lymphocytes of thymomas, although the expression was mainly confirmed on cell membrane and cytoplasm of tumor epithelial cells. High PD-L1 expression was associated with younger age, higher stage, incomplete resection, and WHO histological classification. When adjusted for age and sex, high PD-L1 was correlated with poor overall survival. In our study, $54 \%$ of thymomas demonstrated high PD-L1 expression using a clone of EPR1161[2] (Abcam, Cambridge, UK), with determination of optimal cutoff value using Youden's index (28). Apparent PD-L1 expression identical to that of cytokeratin was determined in our study. High PD-L1 expression was associated with WHO type $\mathrm{B} 2 / \mathrm{B} 3$ and Masaoka stage III/IV disease as found in studies using SP142. In cases underwent complete resection, patients with high PD-L1 expression experienced significantly worse disease-free survival rather than those with low expression. Duan et al. found $46 \%$ of thymomas positive for PD-L1 using antibody of ab58810 (catalog number, rabbit polyclonal, Abcam) (29). They stated that nuclear PD-L1 
staining was observed in 4 cases, whereas mainly expressed on the membrane and in cytoplasm of tumor epithelial cells. However, we should be careful that the release of PD-L1 antibodies adopted by us and Duan et al. is currently cancelled due to improperness for quality criteria of supplier, which has to be confirmed by other reliable antibodies. Bagir et al. reported that $82 \%$ of thymomas revealed high $\mathrm{PD}-\mathrm{L} 1$ expression when they used a clone of 27A2 (Acris Antibodies GmbH, Herford, Germany) and cutoff value of $5 \%$ in number of cells (30). Notably, they also identified that $37 \%$ of thymoma induced PD-L1 expression in tumor infiltrating lymphocytes, macrophages, and histiocytes. However, they did not find any correlation between PD-L1 expression and tumor characteristics or survival.

\section{Conclusions}

Versatile analyses of PD-L1 expression in thymomas involving qualified antibodies clearly demonstrated that they highly express PD-L1 on the cell membrane and cytoplasm of tumor epithelial cells, although the results slightly differed among studies. We should decisively recognize that PD-L1 expression on tumor infiltrating immune cells in thymoma has not been sufficiently defined so far, which requires to be done in future studies to finally verify its role in tumor microenvironment. High PD-L1 expression appears to be found in high grade malignancies such as type B2/B3 thymomas or those with advanced stage, predominantly identified using SP142. PD-L1 is potentially increased by chemotherapy, which is probably induced by immunogenic cell death. Survival impact of PD-L1 expression in thymoma may be determined only in the studies which excluded thymic carcinoma patients. Furthermore, PD-L1 prognostic value also could be identified when we analyzed thymomas which were completely resected, distinct from biopsy and incompletely resected cases; however, it is often hard because of the rareness of this tumor.

\section{Acknowledgments}

Funding: None.

\section{Footnote}

Provenance and Peer Review: This article was commissioned by the Guest Editors (Dragana Jovanovic and Semra
Bilaceroglu) for the series "Thymoma" published in Fournal of Thoracic Disease. The article was sent for external peer review organized by the Guest Editors and the editorial office.

Conflicts of Interest: Both authors have completed the ICMJE uniform disclosure form (available at http://dx.doi. org/10.21037/jtd-19-3703). The series "Thymoma" was commissioned by the editorial office without any funding or sponsorship. The authors have no other conflicts of interest to declare.

Ethical Statement: The authors are accountable for all aspects of the work in ensuring that questions related to the accuracy or integrity of any part of the work are appropriately investigated and resolved.

Open Access Statement: This is an Open Access article distributed in accordance with the Creative Commons Attribution-NonCommercial-NoDerivs 4.0 International License (CC BY-NC-ND 4.0), which permits the noncommercial replication and distribution of the article with the strict proviso that no changes or edits are made and the original work is properly cited (including links to both the formal publication through the relevant DOI and the license). See: https://creativecommons.org/licenses/by-nc-nd/4.0/.

\section{References}

1. Ströbel P, Helmreich M, Menioudakis G, et al. Paraneoplastic myasthenia gravis correlates with generation of mature naive CD4(+) T cells in thymomas. Blood 2002;100:159-66.

2. Masaoka A. Staging system of thymoma. J Thorac Oncol 2010;5:S304-12.

3. Safieddine N, Liu G, Cuningham K, et al. Prognostic factors for cure, recurrence and long-term survival after surgical resection of thymoma. J Thorac Oncol 2014;9:1018-22.

4. David SE, Douglas EW, Dara LA, et al. NCCN Guidelines Version 2, 2018. Thymomas and Thymic Carcinomas. Available online: https://www.nccn.org/. Accessed November 6, 2019.

5. Schmitt J, Loehrer PJ. The role of chemotherapy in advanced thymoma. J Thorac Oncol 2010;5:S357-60.

6. Thomas A, Rajan A, Berman A, et al. Sunitinib in patients with chemotherapy-refractory thymoma and thymic carcinoma: an open-label phase 2 trial. Lancet Oncol 
2015;16:177-86.

7. Liang SC, Latchman YE, Buhlmann JE, et al. Regulation of PD-1, PD-L1, and PD-L2 expression during normal and autoimmune responses. Eur J Immunol 2003;33:2706-16.

8. Brown JA, Dorfman DM, Ma FR, et al. Blockade of programmed death-1 ligands on dendritic cells enhances T cell activation and cytokine production. J Immunol 2003;170:1257-66.

9. Pedoeem A, Azoulay-Alfaguter I, Strazza M, et al. Programmed death-1 pathway in cancer and autoimmunity. Clin Immunol 2014;153:145-52.

10. Dong H, Strome SE, Salomao DR, et al. Tumor-associated B7-H1 promotes T-cell apoptosis: a potential mechanism of immune evasion. Nat Med 2002;8:793-800.

11. Wu C, Zhu Y, Jiang J, et al. Immunohistochemical localization of programmed death-1 ligand-1 (PD-L1) in gastric carcinoma and its clinical significance. Acta Histochem 2006;108:19-24.

12. Nomi T, Sho M, Akahori T, et al. Clinical significance and therapeutic potential of the programmed death-1 ligand/ programmed death-1 pathway in human pancreatic cancer. Clin Cancer Res 2007;13:2151-7.

13. Droeser RA, Hirt C, Viehl CT, et al. Clinical impact of programmed cell death ligand 1 expression in colorectal cancer. Eur J Cancer 2013;49:2233-42.

14. Ohigashi Y, Sho M, Yamada Y, et al. Clinical significance of programmed death-1 ligand-1 and programmed death-1 ligand-2 expression in human esophageal cancer. Clin Cancer Res 2005;11:2947-53.

15. Travis WD, Brambilla E, Burke AP, et al. WHO classification of tumours of the lung, pleura, thymus and heart. 4th edition. Lyon: IARC, 2015:183-243.

16. Yokoyama S, Miyoshi H. Thymic tumors and immune checkpoint inhibitors. J Thorac Dis 2018;10:S1509-15.

17. Owen D, Chu B, Lehman AM, et al. Expression patterns, prognostic value, and intratumoral heterogeneity of PDL1 and PD-1 in thymoma and thymic carcinoma. J Thorac Oncol 2018;13:1204-12.

18. Marchevsky AM, Walts AE. PD-L1, PD-1, CD4, CD8 expression in neo-plastic and nonneoplastic thymus. Hum Pathol 2017;60:16-23.

19. Chen Y, Zhang Y, Chai X, et al. Correlation between the expression of PD-L1 and clinicopathological features in patients with thymic epithelial tumors. Biomed Res Int 2018;2018:5830547.

20. Hakiri S, Fukui T, Mori S, et al. Clinicopathologic features of thymoma with the expression of programmed death ligand 1. Ann Thorac Surg 2019;107:418-24.

21. Guleria P, Husain N, Shukla S, et al. PD-L1 immunoexpression assay in thymomas: study of 84 cases and review of literature. Ann Diagn Pathol 2018;34:135-41.

22. Terra SBSP, Mansfield AS, Vrana JA, et al. Heterogeneity of programmed death-ligand 1 expression in thymic epithelial tumours between initial specimen and synchronous or metachronous metastases or recurrences. Histopathology 2019;74:364-7.

23. Katsuya Y, Fujita Y, Horinouchi H, et al. Immunohistochemical status of PD-L1 in thymoma and thymic carcinoma. Lung Cancer 2015;88:154-9.

24. Weissferdt A, Fujimoto J, Kalhor N, et al. Expression of PD-1 and PD-L1 in thymic epithelial neoplasms. Mod Pathol 2017;30:826-33.

25. Arbour KC, Naidoo J, Steele KE, et al. Expression of PD-L1 and other im-munotherapeutic targets in thymic epithelial tumors. PLoS One 2017;12:e0182665.

26. Tiseo M, Damato A, Longo L, et al. Analysis of a panel of druggable gene mutations and of ALK and PD-L1 expression in a series of thymic epithelial tumors (TETs). Lung Cancer 2017;104:24-30.

27. Padda SK, Riess JW, Schwartz EJ, et al. Diffuse high intensity PD-L1 staining in thymic epithelial tumors. J Thorac Oncol 2015;10:500-8.

28. Yokoyama S, Miyoshi H, Nishi T, et al. Clinicopathologic and prognostic implications of programmed death ligand 1 expression in thymoma. Ann Thorac Surg 2016;101:1361-9.

29. Duan J, Liu X, Chen H, et al. Impact of PD-L1, transforming growth factor-beta expression and tumorinfiltrating CD8+ T cells on clinical outcome of patients with advanced thymic epithelial tumors. Thorac Cancer 2018;9:1341-53.

30. Bagir EK, Acikalin A, Avci A, et al. PD-1 and PD-L1 expression in thymic epithelial tumours and non-neoplastic thymus. J Clin Pathol 2018;71:637-41.

31. Rehman JA, Han G, Carvajal-Hausdorf DE, et al. Quantitative and pathologist-read comparison of the heterogeneity of programmed death-ligand 1 (PD-L1) expression in non-small cell lung cancer. Mod Pathol 2017;30:340-9.

32. Lee KS, Kwak Y, Ahn S, et al. Prognostic implication of CD274 (PD-L1) protein expression in tumorinfiltrating immune cells for microsatellite unstable and stable colorectal cancer. Cancer Immunol Immunother 2017;66:927-39.

33. Bellmunt J, Mullane SA, Werner L, et al. Association of 
PD-L1 expression on tumor-infiltrating mononuclear cells and overall survival in patients with urothelial carcinoma. Ann Oncol 2015;26:812-7.

34. Rimm DL, Han G, Taube JM, et al. A prospective, multi-institutional, pathologist-based assessment of 4 immunohistochemistry assays for PD-L1 expression in non-small cell lung cancer. JAMA Oncol 2017;3:1051-8.

35. Katsuya Y, Horinouchi H, Asao T, et al. Expression of programmed death 1 (PD-1) and its ligand (PD-L1) in

Cite this article as: Yokoyama S, Miyoshi H. Comparison of PD-L1 immunohistochemical assays and the significance of PD-L1 expression in thymoma. J Thorac Dis 2020;12(12):75537560. doi: $10.21037 /$ jtd-19-3703 thymic epithelial tumors: impact on treatment efficacy and alteration in expression after chemotherapy. Lung Cancer 2016;99:4-10.

36. Kepp O, Senovilla L, Kroemer G. Immunogenic cell death inducers as anticancer agents. Oncotarget 2014;5:5190-1.

37. Hato SV, Khong A, de Vries IJ, et al. Molecular pathways: the immunogenic effects of platinum-based chemotherapeutics. Clin Cancer Res 2014;20:2831-7. 